\title{
Associations between Dietary Patterns and Inflammatory Markers during Pregnancy: A Systematic Review
}

\author{
Kuan-Lin Yeh ${ }^{1, *}$, Amber Kautz ${ }^{2} \mathbb{D}$, Barbara Lohse ${ }^{3} \mathbb{D}$ and Susan W. Groth ${ }^{1, *}$ \\ 1 School of Nursing, University of Rochester, Rochester, NY 14642, USA \\ 2 School of Medicine and Dentistry, University of Rochester, Rochester, NY 14623, USA; \\ amber_kautz@urmc.rochester.edu \\ 3 Wegmans School of Health and Nutrition, Rochester Institute of Technology, Rochester, NY 14623, USA; \\ balihst@rit.edu \\ * Correspondence: kuanlin_yeh@urmc.rochester.edu (K.-L.Y.); susan_groth@urmc.rochester.edu (S.W.G.); \\ Tel.: +1-585-276-6147 (K.-L.Y.); 1-585-275-8895 (S.W.G.)
}

check for updates

Citation: Yeh, K.-L.; Kautz, A.; Lohse, B.; Groth, S.W. Associations between Dietary Patterns and Inflammatory Markers during Pregnancy: A Systematic Review. Nutrients 2021, 13, 834. https:// doi.org/10.3390/nu13030834

Academic Editor: Claudia Börnhorst

Received: 17 January 2021

Accepted: 25 February 2021

Published: 4 March 2021

Publisher's Note: MDPI stays neutral with regard to jurisdictional claims in published maps and institutional affiliations.

Copyright: (c) 2021 by the authors. Licensee MDPI, Basel, Switzerland. This article is an open access article distributed under the terms and conditions of the Creative Commons Attribution (CC BY) license (https:/ / creativecommons.org/licenses/by/ $4.0 /)$.

\begin{abstract}
Elevated inflammation in pregnancy has been associated with multiple adverse pregnancy outcomes and potentially an increased susceptibility to future chronic disease. How maternal dietary patterns influence systemic inflammation during pregnancy requires further investigation. The purpose of this review was to comprehensively evaluate studies that assessed dietary patterns and inflammatory markers during pregnancy. This review was guided by the Preferred Reporting Items for Systematic Review and Meta-Analyses. Included studies were sourced from EMBASE, PubMed, Web of Science, and Scopus and evaluated using The Quality Assessment Tool for Quantitative Studies. Inclusion criteria consisted of human studies published in English between January 2007 and May 2020 that addressed associations between dietary patterns and inflammatory markers during pregnancy. Studies focused on a single nutrient, supplementation, or combined interventions were excluded. A total of 17 studies were included. Despite some inconsistent findings, maternal diets characterized by a higher intake of animal protein and cholesterol and/or a lower intake of fiber were shown to be associated with certain pro-inflammatory markers (C-reactive protein (CRP), interleukin-6 (IL-6), tumor necrosis factor- $\alpha$ (TNF- $\alpha$ ), IL-8, serum amyloid A (SAA), and glycoprotein acetylation (GlycA)). Future studies that explore a broader range of inflammatory markers in the pregnant population, reduce measurement errors, and ensure adequate statistical adjustment are warranted.
\end{abstract}

Keywords: diet; dietary patterns; inflammation; inflammatory markers; pregnancy

\section{Introduction}

An amplified inflammatory response during pregnancy has been linked to multiple pregnancy complications [1], such as preeclampsia [2], preterm delivery [3], depressive symptoms [4], and recurrent abortion [5]. Maternal inflammation also contributes to programming of fetal metabolic profiles and immune system through epigenetic modifications [6,7]. Systemic inflammation normally occurs during pregnancy [8]. Proinflammatory markers, such as interleukin-6 (IL-6), tumor necrosis factor- $\alpha$ (TNF- $\alpha)[9,10]$, and C-reactive protein (CRP) [11], increase significantly over the course of pregnancy. The immune response is particularly increased at the times of implantation and parturition [12-14] or during periods of excess maternal psychosocial stress [9]. High levels of IL-6 and TNF- $\alpha$ have been positively associated with gestational diabetes mellitus (GDM) [15]. Elevated CRP and IL-8 have been associated with an increased risk of preeclampsia [16]. The activation of inflammasomes, which induce interleukin $1 \beta$ (IL-1 $\beta$ ) secretion, has been linked to maternal obesity, preterm birth, and preeclampsia [13]. Pro-inflammatory cytokines (IL-6, TNF- $\alpha$, IL-8, IL-1 $\beta$ ) have been found to remain elevated or increase in the first month after childbirth [10]. Women with a history of preeclampsia and persistent 
inflammation during postpartum have an increased risk of cardiometabolic disease and neurodegenerative disorders later in life [17].

The immune response is activated as the body attempts to restore homeostasis following an irritant stimulus [18]. If the response is amplified, increased oxidative stress and vasoconstriction may result in adverse pregnancy outcomes [1]. Activated transcription factors (e.g., nuclear factor kappa-B (NF- $\mathrm{kB})$ ) and increased inflammatory cytokines, in turn, amplify inflammatory response and contribute to the development and progression of disease processes $[18,19]$. In the non-pregnant population, chronic inflammation has been shown to contribute to the development of autoimmune disorders [20], endothelial dysfunction [21], cardiometabolic disorders [20,22,23], and increased risk of cardiovascular morbidity and mortality [24]. Pharmacotherapy targeted at IL-1 $\beta$ [25] and TNF- $\alpha$ [24] has been shown to successfully improve cardiovascular and endothelial function, suggesting that controlling the inflammatory response is a potential target for research.

Evidence from global studies has indicated that diet has a crucial role in the prevention of chronic disease and mortality [6,26]. Multiple nutrients and bioactive substances, such as vitamin $\mathrm{E}$, affect the processes of intracellular signaling and the expression of genes involved in inflammation, with consequent reduction of pro-inflammatory cytokine synthesis [19]. For example, plant phenolic compounds enhance immune function [27] and reduce certain enzymes involved in the generation of reactive oxygen species [28]. Polyphenol antioxidants can modulate immune homeostasis and intracellular signaling by inactivating the NF-KB pathway, modulate mitogen-activated protein kinase (MAPK) $[27,29]$, and suppress toll-like receptor (TLR) [28]. Omega-3 polyunsaturated fatty acid attenuates the activation of TLR4, thereby reducing the production of pro-inflammatory mediators [30]. Conversely, increased dietary exposure to saturated fatty acids and free fatty acids trigger the activation of the c-Jun N-terminal kinase (JNK) and IкB kinase- $\beta$ (IKK $\beta$ ) signaling pathways [31,32]. A high-fat diet has been linked to increased intestinal permeability (leaky gut), resulting in an altered gut microbiome [33] and increased lipopolysaccharide (LPS) (bacterial endotoxins) [34], which activate TLR4 [30]. A complex network of signaling pathways is involved in the interaction of dietary and microbial metabolites and the production of inflammatory mediators [18,19].

An increased interest in synergistic interactions among individual nutrients and, ultimately, dietary patterns has emerged [35]. Dietary patterns refer to foods and beverages that are habitually consumed with respect to their amounts, proportions, variety, combinations, and/or frequency [36]. In the non-pregnant population, high intake of fruits and vegetables, such as in the Nordic diet and Mediterranean diet, have been shown to reduce inflammation [37-40] and promote endothelial function [38] and cardiovascular health $[37,41]$. On the other hand, a high intake of saturated fats, added sugars, and simple carbohydrates have been linked to increased inflammatory markers (CRP/high sensitivity (hs)-CRP, IL-6) [42,43] and risk of hypertension [44] and cardiovascular disease $[37,45]$. Adherence to a Mediterranean diet may modulate low-grade inflammation in pregnancy [46]. A randomized clinical trial (RCT) using a combined lifestyle approach demonstrated the positive effects of carbohydrates on hs-CRP concentrations in late pregnancy [47]. Inflammation-targeted nutrition therapy may provide potential prophylaxis, but little is known about which dietary pattern influences systemic inflammation and which inflammatory markers are altered by diet during pregnancy. Therefore, the aim of this review is to synthesize the current empirical evidence concerning the relationship between dietary patterns and the inflammatory response and identify the inflammatory markers associated with dietary patterns during pregnancy.

\section{Materials and Methods}

This review was guided by the Preferred Reporting Items for Systematic Review and Meta-Analyses (PRISMA). Articles were sourced from 4 databases, namely, EMBASE, PubMed, Web of Science, and Scopus. The literature search was undertaken in September 2018 and updated in May 2020. The search strategy was adapted for each database using 
keywords as follows: inflammation OR "inflammatory markers" AND pregnancy OR "pregnant women" AND diet OR "dietary patterns." No restrictions or filters were applied to the search in the title, abstract, or keywords. Two reviewers (K.Y. and A.K.) independently performed the screening of titles and abstracts, followed by full-text assessment as needed.

The screening process involved the following inclusion criteria: (1) pregnant women as the target population, (2) maternal dietary patterns as the predictor or independent variable, (3) peripheral blood markers of inflammation as the outcome or dependent variable, (4) emphasis on dietary patterns rather than single nutrients, (5) publication in a peer-reviewed journal between 2007 and 2020, (6) intervention or observational studies, (7) publication in English, and (8) human studies with full-text only. For multiple publications based on the same study, only the one with the largest sample size was included. Studies on supplementation or dietary exposure combined with other lifestyle interventions were excluded. The reference lists of included articles were carefully reviewed to identify any additional eligible studies. The screening process was conducted using Endnote and Abstrackr software. Data extraction included several key components (i.e., author, country, study design, participant characteristics, sample size, dietary pattern(s), reference period, dietary assessment tool, and the main findings related to the levels of inflammatory markers) that were verified by a second reviewer. All discrepancies were discussed, and a consensus was reached.

The quality of included studies was independently assessed by 2 reviewers (K.Y. and B.L. or A.K.) using the "Quality Assessment Tool for Quantitative Studies" developed by the Effective Public Health Practice Project (EPHPP) [48]. This appraisal tool has a strong rating in methodology and has been widely used in public health research [48]. Studies were evaluated on 8 key components, namely, selection bias, study design, confounders accounted for, blinding, data collection methods, withdrawals and dropouts, intervention integrity, and analysis. If any of the essential information for the EPHPP quality assessment was absent, cited references were reviewed in detail. A global ranking of strong, moderate, or weak was determined on the basis of the first 6 criteria in accordance with the guideline recommendations [48]. Any discrepancies between the two reviewers' data extraction and global ratings, the included articles, and its relevant reference were reassessed and discussed, and a consensus was reached. A third person was approached for the resolution of any unresolved discrepancies.

\section{Results}

A total of 2639 relevant articles from four databases were extracted, including two articles identified through a review of article reference lists. Of those, 983 duplicates were removed, and the titles and abstracts of the remaining 1656 studies were screened for their eligibility on the basis of inclusion and exclusion criteria. Following the screening, an additional 1626 articles that did not meet the inclusion criteria were excluded. Of the remaining 30 studies, 13 were determined ineligible on the basis of a full-text evaluation. A total of 17 articles were included in the present review. The detailed process of article selection based on the PRISMA guidelines is presented in Figure 1.

\subsection{Study Characteristics}

Characteristics of the included studies are outlined in Table 1. Included studies were conducted in 10 countries from 5 continents, namely, Europe, Australia, Asia, and South and North America. Six studies were from the United States [3,49-53], two were from Iran [54,55], and one each was from China [56], Lebanon [57], the United Kingdom [58], Norway [59], Denmark [60], Australia [61], Finland [62], Ireland [63], and Brazil [11]. Among the included studies, four studies were randomized controlled trials [54,58,59,61]. Sample sizes ranged from 32 [54] to 290 [61], and intervention duration ranged from four weeks [54] to five months [58]. Seven studies were prospective cohort designs [3,11,49-52,56] with the sample sizes ranging between 116 [11] to 1808 [52]. Five studies 
were cross-sectional $[53,57,60,62,63]$, with the sample sizes ranging between 100 [62] to 621 [63], and one study was a case-control study with 135 participants [55].

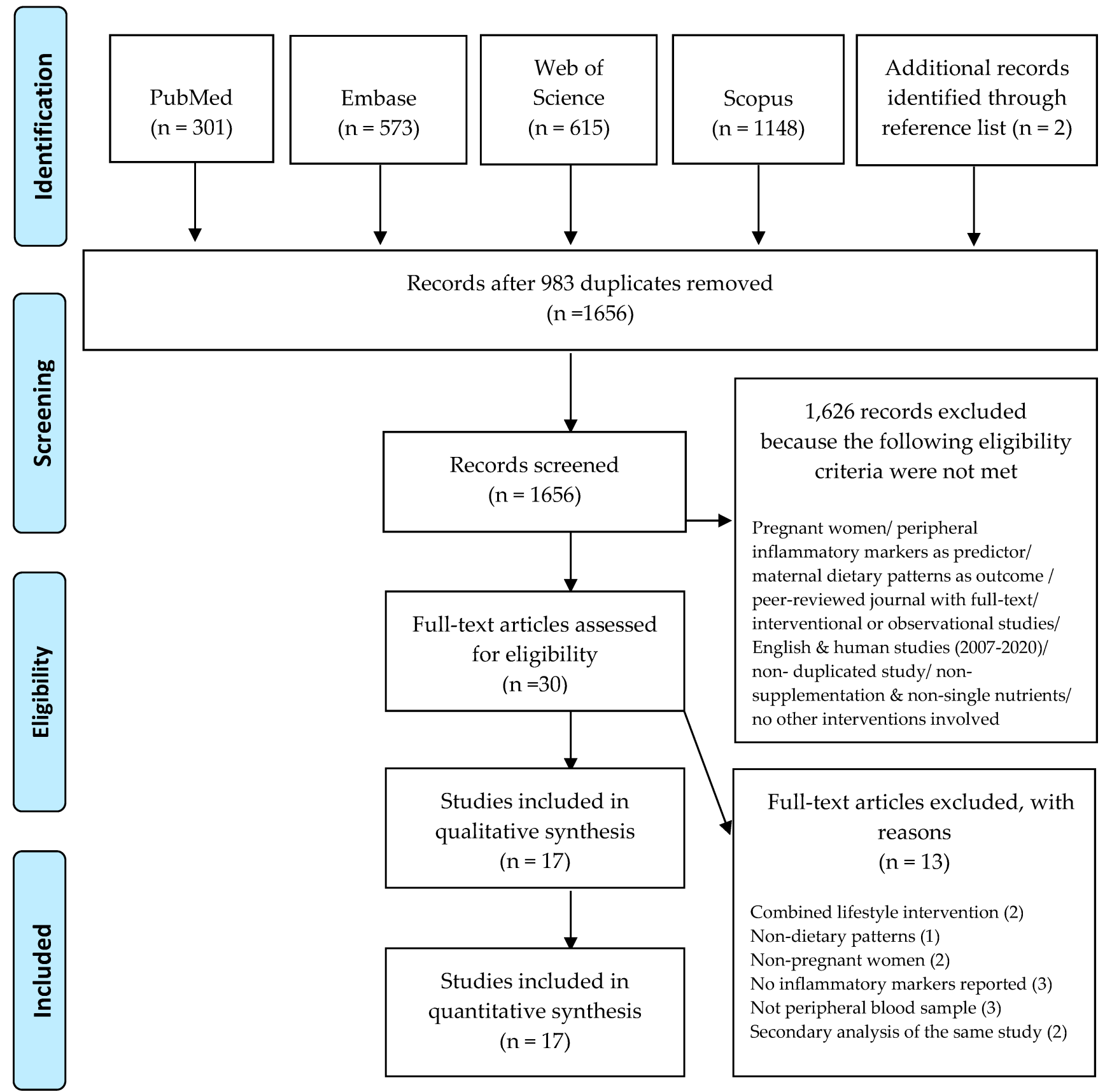

Figure 1. Literature was searched in September 2018 and updated in May 2020. The included studies were published from 2007 to May 2020.

Study samples were diverse and representative of a variety of pregnant populations. Seven studies presented the ethnicity distribution of their study sample [3,49-53,61]. Predominately represented ethnicities included Non-Hispanic white, NonHispanic black, and Hispanic. Participants were between 16 [51] and 45 years of age [55]. The health status of participants varied across studies, including pregnant women who were healthy $[3,11,50,51,56-60]$, healthy but at high risk of GDM [61], healthy but at high risk of giving birth to an infant with macrosomia [63], non-diabetic and with a body mass index (BMI) $18.5 \mathrm{~kg} / \mathrm{m}^{2}$ [52], overweight or obese [62], or who had a history of recurrent miscarriages [55] or GDM [54]. Two studies did not specify participants' pre-existing health 
conditions as part of the enrollment criteria [49,53]. Nine studies described the education distribution of their study sample, indicating the majority of participants had achieved a high school degree or higher [11,49-53,55,61,62].

\subsection{Dietary Patterns}

The observational studies included a priori defined dietary patterns, such as the Mediterranean diet [57], low/high glycemic index (GI) diet, low/high glycemic load (GL) diet [3,11], and pro-/anti-inflammatory diets using the Dietary Inflammatory Index (DII) $[50-53,55,56]$ or Energy-Adjusted Dietary Inflammatory Index (E-DII) [49]. Other dietary patterns were a posteriori derived through a statistical approach with an emphasis on the proportions of macronutrients [40,49,51], fiber [49,51], or cholesterol [51].

Dietary interventions following a priori-defined dietary patterns included the addition of salmon biweekly [58], a low-GI diet [61], an anti-atherogenic diet [59], and the Dietary Approaches to Stop Hypertension (DASH) diet [54]. Common characteristics of these diets included high amounts of fresh plant-based foods (e.g., fruits and vegetables and whole grains) and low intake of processed meats and animal fats [64,65]. These patterns are largely in accordance with the Global Action Plan for the Prevention and Control of Noncommunicable Disease [65].

\subsection{Dietary Assessment}

A variety of dietary assessment tools, including food frequency questionnaires (FFQ), 24-hour dietary recalls, and three-day food diaries/records, were utilized to measure dietary intake. FFQs were used in seven studies $[11,49,52,55,57,58,60]$. The number of items on the FFQs ranged from 100 [58] to 168 [55]. The reference periods of the FFQs varied from the previous 3 months [52,60,66], 3 to 6 months [49], or 12 months [67,68]. The questionnaires were specifically developed for assessing the Mediterranean diet [57], the Middle Eastern diet [69], the Brazilian diet [11], marine n-3 fatty acids intake [60], or the nutrient intake in pregnant Caucasian women [58]. Two were adapted from the Willett questionnaire $[52,55]$. One was developed by the University of Texas MD Anderson Cancer Center [49]. Six studies used 24-hour dietary recalls. Of these, two utilized a single recall [53,57], and four used repeated recalls collected consecutively for three days [56] or non-consecutively at two time-points [3], at three time-points [50,61], or every month during pregnancy [51]. Three-day food diaries/records were used in three studies [54,61,62]. 
Table 1. Characteristics of the studies examining the relationship between diet and inflammatory biomarkers.

\begin{tabular}{|c|c|c|c|c|c|c|c|c|c|}
\hline Study Design & Reference -Cou & nple Size & $\begin{array}{l}\text { Maternal Age } \\
\text { (Years Old) }\end{array}$ & $\begin{array}{l}\text { Health Status and } \\
\text { Gestational Age } \\
\text { at Enrollment }\end{array}$ & $\begin{array}{c}\text { Dietary Exposure } \\
\text { /Control }\end{array}$ & $\begin{array}{c}\text { Dietary } \\
\text { Assessment Tool } \\
\text { (FFQ Reference } \\
\text { Period) } \\
\text { Time of Dietary } \\
\text { Data Collection }\end{array}$ & $\begin{array}{l}\text { Time of Blood } \\
\text { Collection }\end{array}$ & $\begin{array}{c}\text { Examined } \\
\text { Inflammatory } \\
\text { Markers }\end{array}$ & Main Findings \\
\hline \multirow{3}{*}{$\begin{array}{l}\text { Prospective cohort } \\
\text { study }\end{array}$} & $\begin{array}{c}\text { de Oliveira et al., } \\
2015 \\
\text {-Brazil [11] }\end{array}$ & 115 & $20-40$ & $\begin{array}{l}\text { Healthy; } \\
\text { 5-13 weeks of } \\
\text { gestation }\end{array}$ & $\begin{array}{l}\text { Glycemic load } \\
(\mathrm{GL}) \geq \text { median } \\
/ \text { Glycemic load } \\
(\mathrm{GL})<\text { median }\end{array}$ & $\begin{array}{l}\text { A 73-item FFQ (a } \\
\text { 12-month } \\
\text { reference period) } \\
\text { [67] collected at } \\
\text { weeks } 20-26 \text { of } \\
\text { gestation. }\end{array}$ & $\begin{array}{l}\text { (Fasting) blood } \\
\text { sample at } 3 \\
\text { timepoints (weeks } \\
5-13,20-26,30-36 \\
\text { of gestation) }\end{array}$ & CRP & $\begin{array}{c}\text { Dietary glycemic } \\
\text { load was } \\
\text { negatively } \\
\text { associated with } \\
\text { CRP } \\
\text { concentrations ( } \beta \\
=-0.203 ; 95 \% \text { CI, } \\
-0.380 \text { to }-0.026, p \\
=0.025) .\end{array}$ \\
\hline & $\begin{array}{c}\text { Lindsay et al., } \\
\text { 2018 } \\
\text {-USA [50] }\end{array}$ & 202 & $>18$ & $\begin{array}{l}\text { Healthy during } \\
\text { the first or early } \\
\text { second trimester }\end{array}$ & $\begin{array}{c}\text { Anti-/pro- } \\
\text { inflammatory diet }\end{array}$ & $\begin{array}{c}\text { Interviewer } \\
\text { administered } 24 \text {-h } \\
\text { dietary recalls } \\
\text { collected at mean } \\
\text { week } 12.9 \pm 1.7 \\
20.5 \pm 1.4, \text { and } \\
30.4 \pm 1.4 \text { of } \\
\text { gestation. }\end{array}$ & $\begin{array}{c}\text { Day } 6 \text { of each } \\
\text { assessment period } \\
\text { at } 10-12 \text { weeks, } \\
20-22 \text { weeks, } \\
30-32 \text { weeks of } \\
\text { gestation }\end{array}$ & TNF- $\alpha$ & $\begin{array}{c}\text { The DII scores } \\
\text { ranged from }-4.3 \\
\text { to } 3.7 \text { using } 32 \\
\text { food parameters. } \\
\text { DII was positively } \\
\text { associated with } \\
\text { TNF- } \alpha(\beta=0.093 \text {, } \\
95 \% \text { CI: } \\
0.023-0.163, p= \\
0.010) .\end{array}$ \\
\hline & $\begin{array}{c}\text { McCullough et al., } \\
2017 \\
\text {-USA [49] }\end{array}$ & 1057 & $\geq 18$ & $\begin{array}{l}\text { Ethnically diverse; } \\
<12 \text { weeks of } \\
\text { gestation }\end{array}$ & $\begin{array}{c}\text { Anti-/pro- } \\
\text { inflammatory diet }\end{array}$ & $\begin{array}{l}\text { FFQ (a 6-month } \\
\text { reference period) } \\
\text { during } \\
\text { peri-conceptional } \\
\text { stage) at 3-time } \\
\text { points: enrollment } \\
\text { (median 12 } \\
\text { weeks), the second } \\
\text { trimester (diet in } \\
\text { the first trimester), } \\
\text { between } 36 \text { weeks } \\
\text { of gestation to } \\
\text { delivery (diet in } \\
\text { the last } 2 \\
\text { trimesters), and at } \\
\text { delivery as } \\
\text { needed. }\end{array}$ & $\begin{array}{l}\text { The blood sample } \\
\text { was taken before } \\
12 \text { weeks of } \\
\text { gestation }\end{array}$ & $\begin{array}{c}\text { INF } \gamma, \text { IL-12, } \\
\text { IL-17A, IL-1 } \beta \text {, } \\
\text { IL-4, IL-6, and } \\
\text { TNF- } \alpha\end{array}$ & $\begin{array}{c}\text { The E-DII scores } \\
\text { ranged from }-5.00 \\
\text { to } 4.96 \text { using } 27 \\
\text { food parameters. } \\
\text { The correlation } \\
\text { between maternal } \\
\text { E-DII and } \\
\text { circulating } \\
\text { cytokines was not } \\
\text { significant ( } p> \\
0.05) .\end{array}$ \\
\hline
\end{tabular}


Table 1. Cont.

\begin{tabular}{|c|c|c|c|c|c|c|c|c|c|}
\hline Study Design & Reference -Cou & aple Size & $\begin{array}{l}\text { Maternal Age } \\
\text { (Years Old) }\end{array}$ & $\begin{array}{l}\text { Health Status and } \\
\text { Gestational Age } \\
\text { at Enrollment }\end{array}$ & $\begin{array}{l}\text { Dietary Exposure } \\
\text { /Control }\end{array}$ & $\begin{array}{c}\text { Dietary } \\
\text { Assessment Tool } \\
\text { (FFQ Reference } \\
\text { Period) } \\
\text { Time of Dietary } \\
\text { Data Collection }\end{array}$ & $\begin{array}{l}\text { Time of Blood } \\
\text { Collection }\end{array}$ & $\begin{array}{c}\text { Examined } \\
\text { Inflammatory } \\
\text { Markers }\end{array}$ & Main Findings \\
\hline $\begin{array}{l}\text { Prospective cohort } \\
\text { study }\end{array}$ & $\begin{array}{c}\text { Moore et al., } 2018 \\
\text {-USA [51] }\end{array}$ & 511 & $\begin{array}{c}\geq 16 \\
(28 \pm 6)\end{array}$ & $\begin{array}{l}\text { Healthy singleton } \\
\text { pregnancy; } \\
<24 \text { weeks of } \\
\text { gestation }\end{array}$ & $\begin{array}{l}\text { Anti-/pro- } \\
\text { inflammatory diet }\end{array}$ & $\begin{array}{c}\text { One ASA24 per } \\
\text { month. At least } \\
\text { one per } \\
\text { participant. Visits } \\
\text { in early pregnancy } \\
\text { (median } 17 \text { weeks } \\
\text { of gestation), } \\
\text { mid-pregnancy } \\
\text { (median } 27 \text { weeks } \\
\text { of gestation), and } \\
\text { at delivery } \\
\text { (median } 1 \text { day } \\
\text { after delivery) }\end{array}$ & $\begin{array}{l}\text { Blood sample at } 27 \\
\text { weeks of gestation }\end{array}$ & IL-6, hs-CRP & $\begin{array}{c}\text { The DII scores } \\
\text { ranged from }-4.4 \\
\text { to } 4.0 \text { using } 28 \\
\text { food parameters. } \\
\text { For each } 1 \text { unit } \\
\text { increase in the DII, } \\
\text { a } 0.12 \mathrm{mg} / \mathrm{L} \\
\text { increase in IL-6 } \\
\text { levels was } \\
\text { detected at } 27 \\
\text { weeks of gestation } \\
\text { (95\% CI, } 0.01-0.24 \text {; } \\
p=0.03) \text {. No } \\
\text { association was } \\
\text { observed with } \\
\text { hs-CRP }(p=0.27) \text {. }\end{array}$ \\
\hline
\end{tabular}


Table 1. Cont.

\begin{tabular}{|c|c|c|c|c|c|c|c|c|c|}
\hline Study Design & Reference -Cou & nple Size & $\begin{array}{c}\text { Maternal Age } \\
\text { (Years Old) }\end{array}$ & $\begin{array}{l}\text { Health Status and } \\
\text { Gestational Age } \\
\text { at Enrollment }\end{array}$ & $\begin{array}{l}\text { Dietary Exposure } \\
\text { /Control }\end{array}$ & $\begin{array}{c}\text { Dietary } \\
\text { Assessment Tool } \\
\text { (FFQ Reference } \\
\text { Period) } \\
\text { Time of Dietary } \\
\text { Data Collection }\end{array}$ & $\begin{array}{l}\text { Time of Blood } \\
\text { Collection }\end{array}$ & $\begin{array}{c}\text { Examined } \\
\text { Inflammatory } \\
\text { Markers }\end{array}$ & Main Findings \\
\hline \multirow[b]{2}{*}{$\begin{array}{l}\text { Prospective cohort } \\
\text { study }\end{array}$} & $\begin{array}{l}\text { Sen et al., } 2016 \\
\text {-USA [52] }\end{array}$ & 1808 & $32.2 \pm 5.0$ & $\begin{array}{c}\text { Non-diabetic at } \\
\text { median } 9.9 \text { weeks } \\
\text { of gestation }\end{array}$ & $\begin{array}{l}\text { Anti-/pro- } \\
\text { inflammatory diet }\end{array}$ & $\begin{array}{l}\text { A 146-item FFQ } \\
\text { and } 33 \text { items for a } \\
\text { supplement intake } \\
\text { assessment } \\
\text { [71] (last } \\
\text { menstrual period } \\
\text { for the first } \\
\text { trimester or } \\
\text { previous three } \\
\text { months during the } \\
\text { second trimester). }\end{array}$ & $\begin{array}{c}(\mathrm{GCT}) \text { at weeks } \\
22-31 \text { of gestation }\end{array}$ & CRP & $\begin{array}{l}\text { The DII scores } \\
\text { ranged from }-5.4 \\
\text { to } 3.7 \text { using } 28 \\
\text { food parameters. } \\
\text { Higher DII was } \\
\text { associated with } \\
\text { higher plasma } \\
\text { CRP in the second } \\
\text { trimester }(\beta=0.08 \text {, } \\
95 \% \text { CI: } 0.02-0.14) .\end{array}$ \\
\hline & $\begin{array}{l}\text { Yang et al., } 2020 \\
\text {-China [56] }\end{array}$ & 307 & $28.5 \pm 3.4$ & $\begin{array}{l}\text { Healthy with } \\
\text { normal BMI; } \\
\text { 16-20 weeks of } \\
\text { gestation }\end{array}$ & $\begin{array}{l}\text { Anti-/pro- } \\
\text { inflammatory diet }\end{array}$ & $\begin{array}{l}\text { Dietary recall for } \\
\text { three consecutive } \\
\text { days since the } \\
\text { date of enrollment } \\
\text { around } 16-20 \\
\text { weeks of } \\
\text { pregnancy. }\end{array}$ & $\begin{array}{c}\text { (Fasting) after } \\
\text { dietary } \\
\text { assessment in the } \\
\text { second trimester }\end{array}$ & hs-CRP & $\begin{array}{c}\text { The odds of } \\
\text { having high levels } \\
\text { of hs-CRP in } \\
\text { pro-inflammatory } \\
\text { diet group were } \\
1.89 \text { times greater } \\
\text { than the odds of } \\
\text { having high levels } \\
\text { of hs-CRP in the } \\
\text { anti-inflammatory } \\
\text { diet group (95\% } \\
\text { CI: } 1.05,3.42, p= \\
0.043) .\end{array}$ \\
\hline $\begin{array}{c}\text { Randomized } \\
\text { controlled clinical } \\
\text { trial }\end{array}$ & $\begin{array}{l}\text { Asemi et al., } 2013 \\
\text {-Iran [54] }\end{array}$ & 32 & $18-40$ & $\begin{array}{l}\text { GDM; } \\
\text { 24-28 weeks of } \\
\text { gestation }\end{array}$ & $\begin{array}{c}\text { DASH } \\
\text { /based on } \\
\text { recommended } \\
\text { acceptable dietary } \\
\text { intake for GDM }\end{array}$ & $\begin{array}{c}\text { Three-day food } \\
\text { diaries throughout } \\
\text { 4-week } \\
\text { intervention } \\
\text { period (24-28 } \\
\text { weeks of } \\
\text { gestation). }\end{array}$ & $\begin{array}{l}\text { (Fasting) blood } \\
\text { sample at } 24-28 \\
\text { weeks of gestation } \\
\text { and after } 4 \text {-week } \\
\text { intervention } \\
\text { period }\end{array}$ & hs-CRP & $\begin{array}{c}\text { Difference in } \\
\text { mean change of } \\
\text { serum hs-CRP } \\
\text { between DASH } \\
\text { and control diet } \\
\text { was not significant } \\
(p>0.05) .\end{array}$ \\
\hline
\end{tabular}


Table 1. Cont.

\begin{tabular}{|c|c|c|c|c|c|c|c|c|c|}
\hline Study Design & Reference -Cou & ple Size & $\begin{array}{l}\text { Maternal Age } \\
\text { (Years Old) }\end{array}$ & $\begin{array}{l}\text { Health Status and } \\
\text { Gestational Age } \\
\text { at Enrollment }\end{array}$ & $\begin{array}{l}\text { Dietary Exposure } \\
\text { /Control }\end{array}$ & $\begin{array}{c}\text { Assessment Tool } \\
\text { (FFQ Reference } \\
\text { Period) } \\
\text { Time of Dietary } \\
\text { Data Collection }\end{array}$ & $\begin{array}{l}\text { Time of Blood } \\
\text { Collection }\end{array}$ & $\begin{array}{l}\text { Examined } \\
\text { Inflammatory } \\
\text { Markers }\end{array}$ & Main Findings \\
\hline \multirow{3}{*}{$\begin{array}{l}\text { Randomized } \\
\text { controlled clinical } \\
\text { trial }\end{array}$} & $\begin{array}{c}\text { Garcia-Rodriguez } \\
\text { et al., } 2012 \\
\text {-United Kingdom } \\
\text { [58] }\end{array}$ & 123 & $18-40$ & $\begin{array}{l}\text { Healthy; } \\
<19 \text { weeks of } \\
\text { gestation }\end{array}$ & $\begin{array}{l}\text { Twice a week (150 } \\
\text { g/portion) of } \\
\text { salmon } \\
\text { /habitual diet low } \\
\text { in oily fish }\end{array}$ & $\begin{array}{c}\text { A 100-item FFQ (a } \\
\text { 12-week reference } \\
\text { period) [66] } \\
\text { collected at weeks } \\
19 / 20 \text { and } 34 \text { of } \\
\text { gestation; a diary } \\
\text { between week } 20 \\
\text { of gestation to } \\
\text { delivery. }\end{array}$ & $\begin{array}{c}\text { (Fasting) blood } \\
\text { sample at week 20, } \\
\text { weeks 32-34 of } \\
\text { gestation and at } \\
\text { week } 38 \text { of } \\
\text { gestation }\end{array}$ & $\begin{array}{c}\text { IL-8, IL-6, TNF- } \alpha \text {, } \\
\text { HGF, NGF, MCP-1 } \\
\text { sCAMs (E-selectin, } \\
\text { ICAM-1, } \\
\text { VCAM-1) }\end{array}$ & $\begin{array}{c}\text { Inflammatory and } \\
\text { vascular } \\
\text { homeostasis } \\
\text { biomarkers were not } \\
\text { affected by the } \\
\text { intake of farmed } \\
\text { salmon }(p>0.05) \text {. }\end{array}$ \\
\hline & $\begin{array}{c}\text { Khoury et al., } 2007 \\
\text {-Norway [59] }\end{array}$ & 290 & $21-38$ & $\begin{array}{l}\text { Healthy, } \\
\text { non-smoking; } \\
\text { 17-20 weeks of } \\
\text { gestation }\end{array}$ & $\begin{array}{l}\text { Anti-atherogenic } \\
\text { diet (low in } \\
\text { saturated fat and } \\
\text { cholesterol) } \\
\text { / usual diet }\end{array}$ & $\begin{array}{l}\text { Weighed } \\
\text { recordings of } \\
\text { intake from } \\
\text { baseline weeks } \\
\text { 17-20 to week } 36 \\
\text { of gestation. }\end{array}$ & $\begin{array}{l}\text { Blood sample at } \\
\text { weeks } 17-20 \text {, week } \\
24 \text {, week } 30 \text {, and } \\
\text { week } 36 \text { of } \\
\text { gestation }\end{array}$ & $\begin{array}{l}\text { hs-CRP, sCAMs } \\
\text { (sVCAM-1, } \\
\text { sICAM-1 and } \\
\text { E-selectin) }\end{array}$ & $\begin{array}{l}\text { None of the } \\
\text { biomarkers were } \\
\text { influenced by the } \\
\text { intervention (low } \\
\text { saturated fat/low } \\
\text { cholesterol diet) } \\
\text { compared to the } \\
\text { control group (usual } \\
\text { diet) }(p>0.05) .\end{array}$ \\
\hline & $\begin{array}{l}\text { Markovic et al., } \\
2016 \text { (GI baby3) } \\
\text {-Australia [61] }\end{array}$ & 139 & $>18$ & $\begin{array}{l}\text { At high risk of } \\
\text { GDM; } \\
\text { 12-20 weeks of } \\
\text { gestation }\end{array}$ & $\begin{array}{l}\text { Low glycemic } \\
\text { index (LGI) } \\
\text { /high-fiber, } \\
\text { moderate GI diet }\end{array}$ & $\begin{array}{l}\text { Two 3-day food } \\
\text { records collected } \\
\text { at weeks } 14-20 \\
\text { and } 36 \text { of gestation } \\
\text { and three 24-hour } \\
\text { dietary recalls as } \\
\text { dietary } \\
\text { compliance } \\
\text { measure collected } \\
\text { at weeks } 18-24, \\
22-28,26-32 \text { of } \\
\text { gestation; five } \\
\text { dietary } \\
\text { consultations from } \\
\text { weeks } 14-20 \\
\text { through } 34-36 \text { of } \\
\text { gestation. }\end{array}$ & $\begin{array}{c}\text { (Fasting) at mean } \\
\text { week } 17.4 \pm 2 \text { and } \\
\text { at week } 36 \text { of } \\
\text { gestation }\end{array}$ & CRP & $\begin{array}{l}\text { Difference in CRP } \\
\text { between the low } \\
\text { glycemic diet group } \\
\text { and high fiber group } \\
\text { was not significant } \\
\text { at the end of the } \\
\text { intervention period } \\
\quad(p>0.05) .\end{array}$ \\
\hline
\end{tabular}


Table 1. Cont.

Study Design $\quad$ Reference -Country/Sample Size $\quad \begin{gathered}\text { Maternal Age } \\ \text { (Years Old) }\end{gathered} \quad \begin{gathered}\text { Health Status and } \\ \text { Gestational Age } \\ \text { at Enrollment }\end{gathered} \quad \begin{gathered}\text { Dietary Exposure } \\ \text { /Control }\end{gathered}$

$\begin{array}{ccc}\begin{array}{c}\text { Dietary } \\ \text { Assessment Tool } \\ \text { (FFQ Reference } \\ \text { Period) }\end{array} & \begin{array}{c}\text { Time of Blood } \\ \text { Collection }\end{array} & \begin{array}{c}\text { Examined } \\ \text { Inflammatory }\end{array} \\ \begin{array}{c}\text { Time of Dietary } \\ \text { Data Collection }\end{array} & & \text { Markers }\end{array}$

\section{Main Findings}

Data Collection

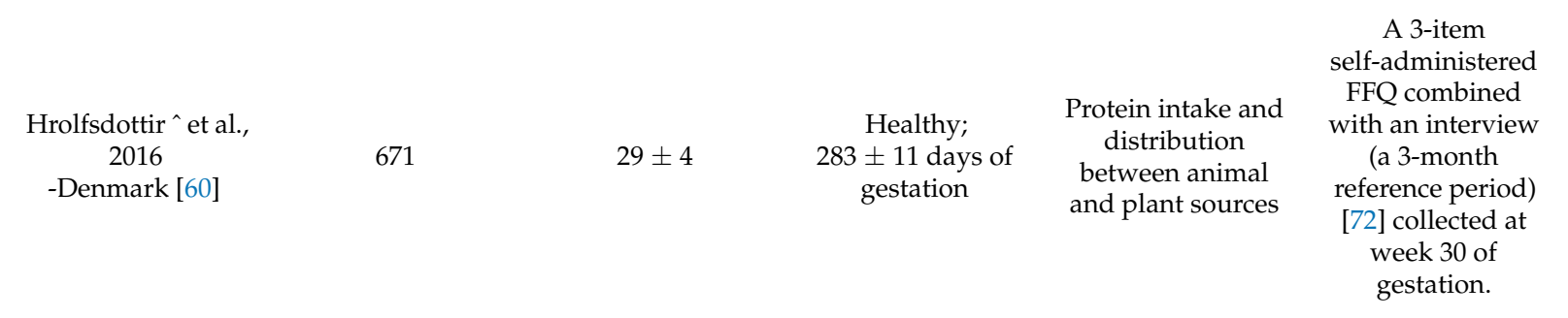
Blood sample at week 30 of gestation hs-CRP, SAA, IL-6, (n) Women in the highest compared to the lowest quintile of animal protein intake had 25\% (95\% CI: $2-53$, $p=0.004$ ) higher hsCRP
hs.004) high

concentrations. A

similar pattern

was observed for

SAA $(23 \%, 95 \%$ CI: $4-47, p=0.003$ ).

Fiber intake was inversely

associated with

IL-8 $(-24 \%, 95 \%$ 0.028).
.

$\begin{array}{ll}\text { A 157-item FFQ } & \\ \text { (12 categories of } & \\ \text { food groups; } & \text { (Fasting) blood } \\ \text { unspecified } & \text { collection in the } \\ \text { reference period) } & \text { second trimester } \\ \text { and one 24-hour } & \text { of pregnancy } \\ \text { recall collected } & \text { otween } 14 \text { and } 27 \\ \text { beeks of } & \\ \text { gestation. } & \end{array}$

lower CRP levels. MFP (OR: 0.90, 95\% CI: 0.82-0.99,

Papazian et al., 2019

100

18-40

Healthy singleton

pregnancy;
14-27 weeks

Mediterranean

gestation diet adherence
CRP

$0.78-0.99, p=0.04$

Med Diet Score

(OR: 0.88, 95\% CI:

(OR. $0.88,95 \% \mathrm{CI}$

$0.80-0.98, p=0.02)$

95\%CI: 0.83-0.99, 
Table 1. Cont.

\begin{tabular}{|c|c|c|c|c|}
\hline Study Design & Reference -Country/Sample Size & $\begin{array}{l}\text { Maternal Age } \\
\text { (Years Old) }\end{array}$ & $\begin{array}{l}\text { Health Status and } \\
\text { Gestational Age } \\
\text { at Enrollment }\end{array}$ & $\begin{array}{l}\text { Dietary Exposure } \\
\text { /Control }\end{array}$ \\
\hline
\end{tabular}

\begin{tabular}{|c|c|c|c|}
\hline $\begin{array}{c}\text { Roytio et al. \#, } \\
2017 \\
\text {-Finland [62] }\end{array}$ & 100 & $30.1 \pm 4.7$ & $\begin{array}{c}\text { Overweight and } \\
\text { obese; } \\
\leq 17 \text { weeks of } \\
\text { gestation }\end{array}$ \\
\hline
\end{tabular}

Three groups: low fiber/moderate fat$$
\text { ; high }
$$

ber/moderate

fat

low fiber/high fat
One 3-day food diary per

participant within

the week before gestation

the study visit.

\section{Time of Blood}

Collection
Examined

Inflammatory

Markers

Main Findings

Recommended dietary intake (DRI) of total fat and fiber was associated with lower levels of GlycA. Correlation between fibelation between fiber total and GlycA were significant $(r=0.316$ $p=0.01)$. No association was found between microbiota or intakes of nutrients and hs-CRP ( $p>$

$$
\text { 0.05) }
$$

The DII scores ranged from -4.98 to 4.14 using 27 food parameters. DII was not associated with CRP $(p>0.05)$. CRP $(p>0.05)$.

\begin{tabular}{|c|c|c|c|c|c|c|}
\hline $\begin{array}{l}\text { Shin et al., } 2017 \\
\text {-USA [53] }\end{array}$ & 561 & $\begin{array}{c}20-44 \\
{[73]}\end{array}$ & $\begin{array}{l}\text { NHANES samples } \\
\text { at mean } 5.35 \pm 0.4 \\
\text { months of } \\
\text { gestation }\end{array}$ & $\begin{array}{l}\text { Anti-/pro- } \\
\text { inflammatory diet }\end{array}$ & $\begin{array}{l}\text { Single 24-hour } \\
\text { dietary recall i } \\
\text { the second }\end{array}$ & $\begin{array}{l}\text { Blood sample in } \\
\text { the second } \\
\text { trimester }\end{array}$ \\
\hline
\end{tabular}

CRP

obese before

pregnancy had

increased odds for being in the highest tertile of the DII and highest tertile of CRP concentration CRP concentration with normal weight.
w 
Table 1. Cont.

Study Design $\quad$ Reference -Country/Sample Size

Study Design $\quad$ Reference -Country/Sample Size $\quad \begin{gathered}\text { Maternal Age } \\ \text { (Years Old) }\end{gathered} \begin{gathered}\text { Gestational Age } \\ \text { at Enrollment }\end{gathered}$ /Control

\section{Table 1. Cont.}

$\begin{array}{ccc}\text { Maternal Age } & \begin{array}{c}\text { Health Status and } \\ \text { Gestational Age } \\ \text { at Enrollment }\end{array} & \begin{array}{c}\text { Dietary Exposure } \\ \text { /Control }\end{array} \\ & \text { ald) }\end{array}$

Dietary

Assessment Tool

FFQ Reference

Period)

Time of Dietary

$\begin{array}{cc}\text { Time of Blood } & \text { Examined } \\ \text { Collection } & \text { Inflammatory } \\ \text { Markers }\end{array}$

\section{Main Findings}

Data Collection

\begin{tabular}{|c|c|c|c|c|c|c|c|}
\hline Cross-sectional & $\begin{array}{l}\text { Walsh et al. *, } 2014 \\
\text {-Ireland [63] }\end{array}$ & 621 & $\geq 18$ & $\begin{array}{c}\text { Secondigravid } \\
\text { whose baby had } \\
\text { macrosomia (birth } \\
\text { weight }>4000 \mathrm{~g} \text { ) } \\
{[74]}\end{array}$ & $\begin{array}{l}\text { Low glycemic } \\
\text { index (LGI) advice } \\
\text { /not receiving LGI } \\
\text { advice }\end{array}$ & $\begin{array}{l}\text { A five-point } \\
\text { Likert-type scale } \\
\text { as an adherence } \\
\text { measure at week } \\
34 \text { of gestation. }\end{array}$ & $\begin{array}{c}\text { (Fasting) blood } \\
\text { sample at mean } \\
\text { week } 13.8 \pm 2.4 \\
\text { and } 28 \text { weeks of } \\
\text { gestation }\end{array}$ \\
\hline
\end{tabular}

Differences between

those who did and those who did not receive low-GI dietary advice were not significant with respect to the concentrations of serum TNF- $\alpha$ or IL-6
in early pregnancy (prior to the intervention) or at 28 weeks of gestation $(p>0.05)$. The DII scores ranged from -0.50 to 2.70 using 31 food parameters. For every 1 unit increase

$$
\begin{aligned}
& \text { in DII, a } \\
& \text { corresponding }
\end{aligned}
$$

With a history of 3 or more miscarriages afte
week 20 of week 20 of
gestation
Anti-/proinflammatory diet
A 168-item FFQ (a 12-month

[68]).
Unspecified

IL-6, CRP

corresponding increase in IL-6 by $0.15 \mathrm{pg} / \mathrm{mL}$ was
detected $(95 \% \mathrm{CI}$ : $<0.01-0.28, p=0.04$ ) no significant association was observed with CRP

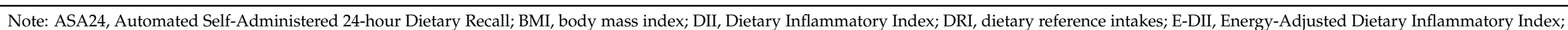

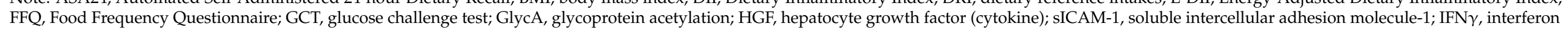

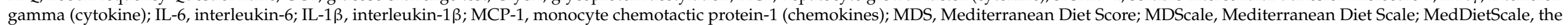

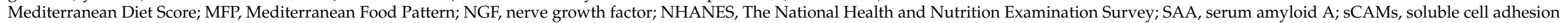

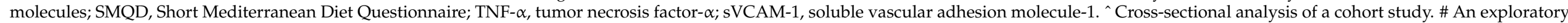
analysis of an observational study. ${ }^{*}$ Secondary analysis of a randomized controlled trial. 
Eight of the studies included only a single dietary measure to assess maternal dietary patterns in the first trimester [62], the second trimester [53-56,60], or both the first and second trimester $[11,57]$. A total of eight studies included repeated dietary measures during the first half of pregnancy [3,52], the second half of pregnancy [58,59,61], or throughout the entire pregnancy [49-51]. Dietary compliance was tracked in the intervention studies with a self-reported five-point Likert adherence scale [63], a fish consumption diary [58], multiple 24-hour dietary recalls [61], or a weighted dietary measure on a pre-determined day every week [59]. Notably, nearly half of the studies [3,50,51,56,57,60,62,63] did not include an assessment or estimation of dietary supplement intake, which is essential to estimate total nutrient intake.

\subsection{Peripheral Markers of Inflammation}

The most frequently exmined inflamatory markers in relation to diet were CRP $[11,52,53,55,57,61] /$ hs-CRP $[3,51,54,56,59,60,62]$ and cytokines (cell signaling molecules) including TNF- $\alpha[49,50,58,63]$, IL-6 $[49,51,55,58,63]$, and IL-8 [58,60]. Less explored cytokines were IL-1 $\beta$, IL-4, IL-17A, IL-12p70, interferon gamma (IFN $\gamma$ ) [49], hepatocyte growth factor (HGF), and monocyte chemotactic protein-1 (MCP-1) - a subfamily of cytokines [58]. Soluble cell adhesion molecules (sCAMS) included vascular adhesion molecule-1 (sVCAM1), intercellular adhesion molecule-1 (sICAM-1), and E-selectin [58,59]. Other reported inflammatory markers were glycoprotein acetylation (GlycA; a novel low-grade inflammatory marker) [62], serum amyloid A (SAA; an acute-phase protein) [60], and nerve growth factor (NGF; neuroinflammatory markers) [58]. Blood specimens were drawn from pregnant women in a fasted state [11,54,56-58,61-63] or during glucose screening tests [3,52]. A variety of biochemical analysis techniques were used in detecting and quantifying inflammatory markers, including enzyme-linked immunosorbent assay (ELISA) [3,54,57,63], nephelometry [53], immunoturbidimetry [11,51,56], and multiplexed method $[49,51,52,58,60]$.

\subsection{Risk of Bias Assessment}

The quality assessment of the included studies is displayed in Table 2. Strong ratings in selection bias were given to studies with participation rates above $80 \%[3,54]$; weak ratings were given to studies with participation rates less than $60 \%$ [61,62]. Only RCTs were rated as strong in study design. Randomization was performed using a random number table $[58,59,66]$ or computer software program $[54,61,63,74]$. For confounders, strong ratings were given to RCTs with groups balanced at baseline $[54,58,59,61]$, the cross-sectional analysis of an RCT [63], and eight observational studies [3,11,49-53,60] that controlled for a number of possible confounding variables (e.g., age, BMI, ethnicity, education, income, parity, smoking). Moderate ratings were given to two observational studies that did not control for smoking status [55,56], which is a notable confounding variable. Two cross-sectional studies that controlled for less than $60 \%$ of confounders $[57,62]$ were identified as weak. A few studies addressed anti-inflammatory medication use [62] and acute inflammatory conditions [11,50,55,57]. 
Table 2. Quality of the included studies assessed by the quality assessment tool for quantitative studies.

\begin{tabular}{|c|c|c|c|c|c|c|c|c|}
\hline Study Design & First Author, Year & 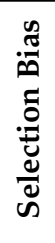 & 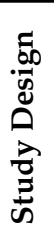 & 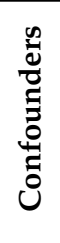 & 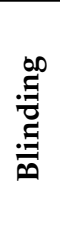 & 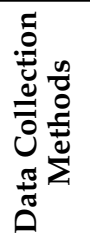 & 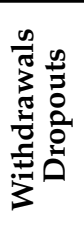 & $\begin{array}{l}\text { Global } \\
\text { Rating }\end{array}$ \\
\hline \multirow{7}{*}{$\begin{array}{l}\text { Prospective } \\
\text { cohort studies }\end{array}$} & de Oliveira et al., 2015 [11] & $\mathrm{m}$ & $\mathrm{m}$ & $\mathrm{s}$ & $\mathrm{w}$ & $\mathrm{m}$ & $\mathrm{s}$ & Moderate \\
\hline & Lindsay et al., 2018 [50] & $\mathrm{m}$ & $\mathrm{m}$ & $\mathrm{s}$ & $\mathrm{w}$ & $\mathrm{s}$ & $\mathrm{m}$ & Moderate \\
\hline & McCullough et al., 2017 [49] & $\mathrm{m}$ & $\mathrm{m}$ & $\mathrm{s}$ & $\mathrm{w}$ & $\mathrm{s}$ & $\mathrm{s}$ & Moderate \\
\hline & Moore et al., 2018 [51] & $\mathrm{m}$ & $\mathrm{m}$ & $\mathrm{s}$ & $\mathrm{w}$ & $\mathrm{s}$ & $\mathrm{m}$ & Moderate \\
\hline & Scholl et al., 2011 [3] & $\mathrm{s}$ & $\mathrm{m}$ & $\mathrm{s}$ & $\mathrm{w}$ & $\mathrm{s}$ & $\mathrm{m}$ & Moderate \\
\hline & Sen et al., 2016 [52] & $\mathrm{m}$ & $\mathrm{m}$ & $\mathrm{s}$ & $\mathrm{w}$ & $\mathrm{s}$ & $\mathrm{s}$ & Moderate \\
\hline & Yang et al., 2020 [56] & $\mathrm{m}$ & $\mathrm{m}$ & $\mathrm{m}$ & $\mathrm{w}$ & $\mathrm{s}$ & $\mathrm{s}$ & Moderate \\
\hline \multirow{4}{*}{$\begin{array}{l}\text { Intervention } \\
\text { studies }\end{array}$} & Asemi et al., 2013 [54] & $\mathrm{s}$ & $\mathrm{s}$ & $\mathrm{s}$ & $\mathrm{s}$ & $\mathrm{s}$ & $\mathrm{s}$ & Strong \\
\hline & $\begin{array}{c}\text { Garcia-Rodriguez et al., } \\
2012 \text { [58] }\end{array}$ & $\mathrm{m}$ & $\mathrm{s}$ & $\mathrm{s}$ & $\mathrm{m}$ & $\mathrm{m}$ & $\mathrm{s}$ & Strong \\
\hline & Khoury et al., 2007 [59] & $\mathrm{m}$ & $\mathrm{s}$ & $\mathrm{s}$ & $\mathrm{m}$ & $\mathrm{w}$ & $\mathrm{m}$ & Moderate \\
\hline & Markovic et al., 2016 [61] & $\mathrm{w}$ & $\mathrm{s}$ & $\mathrm{s}$ & $\mathrm{m}$ & $\mathrm{s}$ & $\mathrm{s}$ & Moderate \\
\hline \multirow{5}{*}{$\begin{array}{l}\text { Cross-sectional } \\
\text { studies }\end{array}$} & Hrolfsdottir^ et al., 2016 [60] & $\mathrm{m}$ & $\mathrm{m}$ & $\mathrm{s}$ & $\mathrm{w}$ & $\mathrm{m}$ & $\mathrm{m}$ & Moderate \\
\hline & Papazian et al., 2019 [57] & $\mathrm{m}$ & $\mathrm{m}$ & $\mathrm{w}$ & $\mathrm{w}$ & $\mathrm{s}$ & $\mathrm{s}$ & Weak \\
\hline & Roytio \# et al., 2017 [62] & $\mathrm{w}$ & $\mathrm{m}$ & $\mathrm{w}$ & $\mathrm{w}$ & $\mathrm{s}$ & $\mathrm{s}$ & Weak \\
\hline & Shin et al., 2017 [53] & $\mathrm{m}$ & $\mathrm{m}$ & $\mathrm{s}$ & $\mathrm{s}$ & $\mathrm{m}$ & $\mathrm{m}$ & Strong \\
\hline & Walsh * et al., 2014 [63] & $\mathrm{m}$ & $\mathrm{m}$ & $\mathrm{s}$ & $\mathrm{w}$ & $\mathrm{s}$ & $\mathrm{s}$ & Moderate \\
\hline $\begin{array}{l}\text { Case-control } \\
\text { studies }\end{array}$ & Vahid et al., 2017 [55] & $\mathrm{m}$ & $\mathrm{m}$ & $\mathrm{m}$ & $\mathrm{w}$ & $\mathrm{s}$ & $\mathrm{m}$ & Moderate \\
\hline
\end{tabular}

Notes: Global rating (criteria): strong (no weak rating), moderate (one weak rating), and weak (two or more weak rating) [65]. ^Cross-sectional analysis of a cohort study. \# An exploratory analysis of an observational study. * Secondary analysis of a randomized controlled trial.

A double-blind design in the RCTs was considered strong [54], and a single-blind design moderate $[58,59,61]$. All observational studies, with the exception of the study using the data from National Health and Nutrition Examination Survey (NHANES) [53], were rated as weak for blinding. Strong ratings were given to the studies using standard assessment tools (e.g., Willett FFQ or 24-hour dietary recalls) [3,49-52,55-57,61-63]. Instruments with known validity or reliability are recognized to have good ratings in data collection methods per the EPHPP dictionary [75]. The study using one single 24-hour dietary recall for measuring dietary intake [53] was rated as moderate. The method of using predetermined days for dietary reporting, which is likely to be altered by social disability, was evaluated as weak [59]. Moderate ratings in withdrawals and dropouts were given to studies with a case-control design [55], a study completion rate of less than $80 \%[51,53,60]$, a lack of reporting the reasons for withdrawal [59], or only reporting the number of cases with completed data $[3,50]$. Overall, the majority of the included studies were classified as moderate methodological quality $[3,11,49-52,55,56,58-61,63]$. Two studies were classified as weak quality $[57,62]$, and three studies were classified as strong quality $[53,54,58]$.

\subsection{Dietary Patterns and Inflammatory Markers}

Dietary patterns described in the studies were not uniform and therefore were categorized on the basis of how the pattern was defined (a posteriori-derived vs. a priori-defined) along with the research design. The majority of observational studies $(n=10)$ indicated a statistically significant association $(p<0.05)$ between diet and inflammatory markers 
(CRP/hs-CRP, IL-6, IL-8, TNF- $\alpha$, SAA, or GlycA). However, diet-inflammation associations were not found in the intervention studies.

\subsubsection{Proportions of Protein, Cholesterol, and/or Fiber}

A posteriori-derived approach was used to examine the proportions of macronutrients among the observational studies. Dietary patterns characterized by higher cholesterol and protein intake were associated with increased hs-CRP in the first trimester [3]. Protein from animal sources was associated with higher hs-CRP and SAA in the third trimester [60]. Lower fat (especially saturated fatty acids) and higher fiber intake contributed to greater diversity and richness of the gut microbiome, which was correlated with lower GlycA but not hs-CRP in the first trimester [62]. Dietary intake of fiber was found to be inversely correlated with levels of CRP in the second trimester [57] and IL-8 in the third trimester [60].

\subsubsection{Anti-/Pro-Inflammatory Diet}

In the observational studies, some inconsistencies were found among the studies of pro-inflammatory diets quantified by the DII (the higher the pro-inflammatory potential of diet, the higher the DII). DII was shown to be positively associated with levels of CRP [52]/hs-CRP [56] during the second trimester when repeated dietary measures were used. Conversely, a positive association between DII and hs-CRP in the second trimester was not found using a single 24-hour dietary recall [53]. The associations between DII and CRP/hs-CRP were not statistically significant in the first [55] or third [51] trimester. Certain cytokines were positively associated with DII, including IL-6 in the first [55] and second [51] trimesters and TNF- $\alpha$ across all three trimesters [50]. Notably, the association between DII and TNF- $\alpha$ was only observed in pregnant women with high levels of stress [50]. Inflammatory diets measured by the E-DII were not associated with circulating cytokines (TNF- $\alpha$, IL-6, IL-1b, IL-4, IL-17A, IL-12p70, IFN $\gamma$ ) in early pregnancy [49].

\subsubsection{High/Low GI/GL Diet}

With respect to carbohydrate content and associated glycemic burden, the results were mixed among the observational and intervention studies. In one cohort study, GI was positively associated with hs-CRP among healthy women with a BMI less than $25 \mathrm{~kg} / \mathrm{m}^{2}$; dietary glycemic load (GL), which addresses both the quality and quantity of carbohydrates, was not associated with hs-CRP levels in the first trimester [3]. In contrast, a different cohort study indicated a non-significant association between GI and CRP, but an inverse association between GL and CRP in healthy women throughout pregnancy [11]. An intervention of low GI advice had no impact on levels of CRP in women with a high risk of GDM [61] nor on IL-6 and TNF- $\alpha$ in women at high risk for macrosomia [63] in the second half pregnancy.

3.6.4. Mediterranean Diet, DASH Diet, Anti-Atherogenic Diet, and Omega-3 Fatty Acid-Enriched Diet

Findings from an observational study indicated a significant association between higher adherence to the Mediterranean diet and lower CRP levels in the second trimester [57]. However, an effect of diet on inflammation was not observed in the intervention studies focusing on an anti-atherogenic diet [59], a DASH diet [54], or omega-3 fatty acid-enriched diet [58]. Specifically, an intervention using the DASH diet had no significant effects on levels of hs-CRP in the second trimester [54]. An anti-atherogenic diet had no effect on levels of hs-CRP and sCAMS [59], and regular salmon intake had no effect on IL-8, IL-6, TNF- $\alpha$, HGF, NGF, MCP-1, HGF, or sCAMS in the second half of pregnancy [58].

\section{Discussion}

This systematic review presents evidence from observational studies and controlled trials of relationships between maternal dietary patterns and inflammatory markers. Dietary patterns explored in this review included the pro-/anti-inflammatory diet, low GI/GL 
diet, Mediterranean diet, DASH diet, and an anti-atherogenic diet. Additionally, the macronutrient composition and regular salmon intake, which addressed the proportions or frequency of dietary components, were examined. Overall, about two-thirds of the studies with moderate quality in methodology per EPHPP showed associations between dietary patterns and pro-inflammatory markers; however, the three studies with strong methodology did not report significant associations, including one observational study [53] and two intervention studies [54,58].

\subsection{Evidence from the Observational Studies}

A dietary pattern with high animal protein, high cholesterol, and/or low fiber was significantly associated with higher levels of CRP [52,57,60]/hs-CRP [3,56] and SAA [60] during pregnancy. Lower fiber intake was associated with higher IL-8 [60] and reduced gut microbiota richness, which contributes to higher GlycA [62]. Interestingly, microbiota composition was not correlated to hs-CRP, suggesting a possible different mechanism of inflammation [62]. Diets categorized as low-DII [50-52,55,56] were associated with lower levels of pro-inflammatory markers in pregnancy. Dietary intake with less antiinflammatory food parameters (e.g., fiber, vitamins, $\beta$-carotene, and flavones) and more pro-inflammatory food parameters (e.g., saturated fats, cholesterol) were associated with higher IL-6 [51,55] and TNF- $\alpha$ [50]. Notably, adherence to a Mediterranean diet was correlated with lower CRP [55].

Relationships between dietary GI/GL and inflammatory markers were inconsistent. Inflammation was found to be positively associated with GI [3] and negatively associated with high GL diets [11]. GI ranks carbohydrates according to their effect on blood glucose levels [76]. GL takes both the quality and total amount of carbohydrates into consideration and is a mathematical product of GI and the quantity of carbohydrates consumed [76]. An inverse association between GL, but not GI, and inflammatory markers [11] could be a function of multiple factors. First, the GI and GL values were derived from a FFQ measurement that consisted of broad food groupings [67], increasing the likelihood of misclassification and an underestimation of an association [76]. Second, this particular FFQ was shown to overestimate fruit intake [67], a systematic error that could lead to a biased finding [77]. Third, a low GL diet could be low in carbohydrates but high in saturated fat and protein [76], which are pro-inflammatory [78]. Compared to a high complex carbohydrate/low fat dietary pattern, a conventional diet low in carbohydrates and high in fat was associated with elevated inflammation during pregnancy [79]. Variations in individual insulin response, mixed meals that influence the glycemic response, and food processing techniques are other possible factors affecting GI/GL [76].

The association of DII with inflammatory markers was inconsistent across four studies $[49,51,53,55]$. Higher DII was associated with a higher concentration of CRP/hs-CRP in two studies [52,56] but not replicated in the DII studies that showed a statistically significant association between a pro-inflammatory diet and IL-6 [51,55]. One factor to consider is that IL-6 is an inducer of hepatic CRP synthesis in hepatocytes [80,81]. Given the mediating effects of IL-6 on the production of hs-CRP, the timing of inflammatory marker measurement could influence the results [51]. In addition, DII was associated with TNF- $\alpha$ only in the context of psychological stress. Among the DII studies, two containing notable measurement issues showed a non-significant association between DII and inflammatory markers $[49,53]$. One cross-sectional study using NHANES data was limited by its dietary measurement of a single 24-hour dietary recall [53] to represent usual dietary intake, increasing the risk of exposure misclassification [77]. Another study included a DII that was energy-adjusted to account for variation in the total energy consumption [49]. However, despite collecting multiple dietary assessments throughout pregnancy, blood samples were collected only at enrollment. Given the changing nature of dietary intake and inflammation during pregnancy, corresponding repeated measures are necessary to reduce measurement error. 
Measurement of dietary intake can be challenging. Therefore, the majority of the longitudinal studies used repeated measures of dietary assessments [3,49-52], enabling a better estimation of habitual intake [77]. To improve dietary estimates, differing dietary assessment tools can be used in combination [82]. For example, measurement error was reduced in one investigation of adherence to the Mediterranean diet by using both a FFQ and a 24-hour dietary recall [57]. Other factors that may have influenced associations between dietary patterns and maternal inflammatory markers include confounding factors, such as age, BMI [53,83], parity [11,83], and smoking status [83,84]. Nearly two-thirds $(n=8)$ of the included observational studies adjusted for known confounders. Among the observational studies with a full model adjustment, a significant association between dietary pattern and inflammation was reported $[3,50-52,60]$. However, information regarding the use of anti-inflammatory medication (e.g., nonsteroidal anti-inflammatory drugs) or the occurrence of acute infections (e.g., periodontitis, urinary tract infections) was frequently left out [85]. Additionally, genetic and microbiome profiles that may influence the inflammatory response to nutrients $[85,86]$ were not considered.

\subsection{Evidence from the Intervention Studies}

Causal effects of the low GI diet, DASH diet, anti-atherogenic diet, and regular salmon intake on inflammation during pregnancy were not evident in the examined RCTs. The lack of blinding in many of the studies, although a challenge in dietary intervention research [87], could lead to considerable expectation bias [88]. Unblinded participants could change their behaviors, and unblinded outcome assessors could unintentionally produce biased results, thereby inducing threats to internal validity [88].

The effect of dietary GI on CRP levels in pregnancy was not established [61]. Threeday food records were combined with 24-hour dietary recalls [61] to improve the accuracy of dietary measurement. However, the intervention and control diets were similarly healthy, which reduced variation in dietary exposure and the ability to detect differing effects of the interventions [89]. In addition, this GI study may have been underpowered given that inflammation was neither the primary nor secondary outcome [90]. Of note, the specification of the type of carbohydrate was lacking in this study, decreasing the interpretability of findings. A complex carbohydrate is mainly sourced from unprocessed and whole, plant-based foods [91], whereas simple carbohydrates are often found in highly processed foods [91]. Whole, plant-based foods are generally accepted as anti-inflammatory, whereas ultra-processed foods are considered pro-inflammatory [92].

The DASH diet did not have an effect on hs-CRP concentrations in pregnant women. However, the sample size of this RCT was small $(n=32)$, and groups reported similar amounts of energy and protein intake and high amounts of vegetable and fruit intake over the 4-week study period [54]. Additionally, associations between the omega-3 fatty acid-enriched diet and plasma inflammatory markers were not established [58]. A study that focused on the effects of routine salmon intake on cytokines using combined dietary assessments (a FFQ and a seafood diary [66]) indicated a non-significant result. The authors concluded that omega-3 intake may have been too low ( $<2 \mathrm{~g} /$ day) to reduce inflammation [58].

The anti-atherogenic diet did not have an effect on the levels of hs-CRP and sCAMS during pregnancy [59]. A reduced intake of cholesterol and saturated fat did not have a lowering effect on inflammation, which is contrary to the results of the observational study [3]. This discrepancy may be related to the methodological approach that included a weekly weighted dietary intake on a pre-determined day throughout pregnancy [59]. The predictability of this approach in the intervention study could have influenced participants' dietary behaviors and compliance on the days of reporting their dietary intake. Furthermore, a type II error could have occurred, as a power calculation was not conducted for the outcome of inflammation [59]. 


\subsection{Anti-Inflammatory Markers and Inflammation Resolution}

Few studies in this review addressed anti-inflammatory cytokines in relation to dietary patterns. Only IL-4 [49] and HGF [58] were examined. Specialized pro-resolving mediators (SPMs), which are metabolites of omega-3 fatty acids responsible for resolving inflammation [93], were not explored. Current understanding of the anti-inflammatory properties of omega-3 fatty acids includes not only inhibition of pro-inflammatory signaling molecules but also the involvement of pro-resolving mediators to accelerate inflammation resolution $[94,95]$. Researchers have found that deficiencies in dietary omega-3 fatty acids may result in insufficient precursors of pro-resolving mediators, leading to prolonged inflammation [94]. An anti-inflammatory approach [25] and pro-resolving mediators [95] are emerging therapeutic targets for disease prevention in human research. To date, SPMs have not been explored in studies focusing on maternal dietary patterns. Pro-resolving mediators have only been explored in RCTs that focus on maternal dietary supplementation [96,97].

\subsection{Strengths and Limitations}

To the best of our knowledge, this is the first systematic review investigating dietary patterns and their association with inflammatory markers during pregnancy. The included studies were conducted in different pregnant populations across the globe, increasing the generalizability of findings. However, the associations between dietary patterns and inflammatory markers during pregnancy must be interpreted with caution for three main reasons. First, dietary assessment is a non-exact science and involves measurement problems. Second, variability in study design and rigor are evident, and third, types of dietary patterns and a lack of exactness in their description contribute to a less cohesive finding. Circulating cytokines (e.g., IL-6, IL-8, IL-1 $\beta$, TNF- $\alpha$ ) or CRP alone are non-specific inflammatory markers [90], and a consensus has not yet been reached for which markers are most pertinent or reflective of phase or acuity of inflammation in pregnancy [81]. Additionally, the exclusion of studies published in other languages limits the inclusion of findings reported by nonEnglish speaking populations. Finally, a meta-analysis could not be conducted because of the methodological and statistical heterogeneity of the included studies.

\subsection{Implications for Clinical Practice and Research}

The findings of this review highlight the potential role of maternal dietary patterns to shape the inflammatory response during pregnancy. Consuming certain dietary patterns (e.g., high intake of saturated fats and low intake of fruits and vegetables) may contribute to higher concentrations of inflammatory markers in pregnancy. Anti-inflammatory dietary patterns correspond to healthy patterns of eating, which is consistent with adherence to the current dietary recommendations. Future studies are needed to examine the changes of anti-inflammatory markers and pro-resolving mediators in relation to maternal dietary patterns to improve understanding of dietary effects in the context of pregnancy. In the research design phase, researchers can consider the adjustment of multiple confounding variables, such as psychosocial and lifestyle factors and the use of anti-inflammatory medication. Additional longitudinal studies with robust methodology and large sample sizes are needed to investigate the associations between dietary patterns and inflammatory response among pregnant women.

\section{Conclusions}

Findings from the observational studies, although inconsistent, suggest that dietary patterns may be associated with pro-inflammatory markers, such as CRP, IL-6, IL-8, TNF- $\alpha$, SAA, and GlycA, during pregnancy. Maternal dietary patterns characterized by high intakes of animal protein and cholesterol, and/or low intakes of fiber were associated with higher inflammatory status. However, all of the included intervention studies reported no dietary effects on inflammation. The most commonly studied inflammatory marker in pregnancy is CRP, but only 6 out of 13 studies showed any relationship of CRP with a dietary pattern. Discrepancies in the findings among the assessed studies may be partly because 
of measurement errors and timing of data collection. These discrepancies are reflective of the complexities and unknown ramifications of dietary effects on inflammation during pregnancy. Future longitudinal studies investigating a broader range of inflammationrelated biomarkers throughout pregnancy, coupled with rigorous designs, are warranted.

Author Contributions: Study design, K.-L.Y.; literature search, K.-L.Y. and A.K.; data extraction, K.-L.Y. and A.K.; validation, K.-L.Y., and A.K.; EPHPP quality assessment, K.-L.Y., A.K., and B.L.; writing-original draft preparation, K.-L.Y.; writing-review and editing, A.K., B.L., and S.W.G.; supervision, B.L. and S.W.G. All authors have read and agreed to the published version of the manuscript.

Funding: This research received no external funding.

Institutional Review Board Statement: Not applicable.

Informed Consent Statement: Not applicable.

Data Availability Statement: Not applicable.

Acknowledgments: Our research team would like to thank Hyekyun Rhee, RN, PNP, FAAN, for offering insightful discussion on the scope of the review. We also appreciate the assistance provided by The Center for Academic and Professional Success at the University of Rochester School of Nursing.

Conflicts of Interest: The authors declare no conflict of interest.

\section{References}

1. Cotechini, T.; Graham, C. Aberrant maternal inflammation as a cause of pregnancy complications: A potential therapeutic target? Placenta 2015, 36, 960-966. [CrossRef] [PubMed]

2. Tosun, M.; Celik, H.; Avci, B.; Yavuz, E.; Alper, T.; Malatyalioğlu, E. Maternal and umbilical serum levels of interleukin-6, interleukin-8, and tumor necrosis factor- $\alpha$ in normal pregnancies and in pregnancies complicated by preeclampsia. J. Matern. Neonatal Med. 2010, 23, 880-886. [CrossRef] [PubMed]

3. Scholl, T.O.; Chen, X.; Goldberg, G.S.; Khusial, P.R.; Stein, T.P. Maternal diet, C-reactive protein, and the outcome of preg-nancy. J. Am. Coll. Nutr. 2011, 30, 233. [CrossRef]

4. Cassidy-Bushrow, A.E.; Peters, R.M.; Johnson, D.A.; Templin, T.N. Association of depressive symptoms with inflammatory biomarkers among pregnant African-American women. J. Reprod. Immunol. 2012, 94, 202-209. [CrossRef] [PubMed]

5. Shaarawy, M.; Nagui, A.R. Enhanced expression of cytokines may play a fundamental role in the mechanisms of immunolog-ically mediated recurrent spontaneous abortion. Acta Obstet. Gynecol. Scand. 1997, 76, 205-211. [CrossRef] [PubMed]

6. Furman, D.; Campisi, J.; Verdin, E.; Carrera-Bastos, P.; Targ, S.; Franceschi, C.; Ferrucci, L.; Gilroy, D.W.; Fasano, A.; Miller, G.W.; et al. Chronic inflammation in the etiology of disease across the life span. Nat. Med. 2019, 25, 1822-1832. [CrossRef] [PubMed]

7. Hantsoo, L.; Kornfield, S.; Anguera, M.C.; Epperson, C.N. Inflammation: A Proposed Intermediary Between Maternal Stress and Offspring Neuropsychiatric Risk. Biol. Psychiatry 2019, 85, 97-106. [CrossRef]

8. Palm, M.; Axelsson, O.; Wernroth, L.; Larsson, A.; Basu, S. Involvement of inflammation in normal pregnancy. Acta Obstet. Gynecol. Scand. 2013, 92, 601-605. [CrossRef] [PubMed]

9. Coussons-Read, M.E.; Okun, M.L.; Nettles, C.D. Psychosocial stress increases inflammatory markers and alters cytokine production across pregnancy. Brain Behav. Immun. 2007, 21, 343-350. [CrossRef] [PubMed]

10. Christian, L.M.; Porter, K. Longitudinal changes in serum proinflammatory markers across pregnancy and postpartum: Effects of maternal body mass index. Cytokine 2014, 70, 134-140. [CrossRef] [PubMed]

11. De Oliveira, L.C.; Franco-Sena, A.B.; Rebelo, F.; Farias, D.R.; Lepsch, J.; Lima, N.D.S.; Kac, G. Factors associated with maternal serum C-reactive protein throughout pregnancy: A longitudinal study in women of Rio de Janeiro, Brazil. Nutrients 2015, 31, 1103-1108. [CrossRef]

12. Mor, G.; Cardenas, I.; Abrahams, V.; Guller, S. Inflammation and pregnancy: The role of the immune system at the implantation site. Ann. N.Y. Acad. Sci. 2011, 1221, 80-87. [CrossRef]

13. Khan, R.N.; Hay, D.P. A clear and present danger: Inflammasomes DAMPing down disorders of pregnancy. Hum. Reprod. Updat. 2014, 21, 388-405. [CrossRef] [PubMed]

14. Mor, G.; Cardenas, I. The Immune System in Pregnancy: A Unique Complexity. Am. J. Reprod. Immunol. 2010, 63, 425-433. [CrossRef] [PubMed]

15. Gomes, C.P.; Torloni, M.R.; Alexandre, S.M.; Mattar, R.; Daher, S.; Gueuvoghlanian-Silva, B.Y. Cytokine Levels in Gestational Diabetes Mellitus: A Systematic Review of the Literature. Am. J. Reprod. Immunol. 2013, 69, 545-557. [CrossRef] [PubMed]

16. Black, K.D.; Horowitz, J.A. Inflammatory Markers and Preeclampsia. Nurs. Res. 2018, 67, 242-251. [CrossRef] [PubMed]

17. Cheng, S.-B.; Sharma, S. Preeclampsia and health risks later in life: An immunological link. Semin. Immunopathol. 2016, 38, 699-708. [CrossRef] 
18. Chen, L.; Deng, H.; Cui, H.; Fang, J.; Zuo, Z.; Deng, J.; Li, Y.; Wang, X.; Zhao, L. Inflammatory responses and inflammationassociated diseases in organs. Oncotarget 2017, 9, 7204-7218. [CrossRef] [PubMed]

19. Calder, P.C.; Albers, R.; Antoine, J.-M.; Blum, S.; Bourdet-Sicard, R.; Ferns, G.A.; Folkerts, G.; Friedmann, P.S.; Frost, G.S.; Guarner, F.; et al. Inflammatory Disease Processes and Interactions with Nutrition. Br. J. Nutr. 2009, 101, 1-45. [CrossRef]

20. Ricordi, C.; García-Contreras, M.; Farnetti, S. Diet and Inflammation: Possible Effects on Immunity, Chronic Diseases, and Life Span. J. Am. Coll. Nutr. 2015, 34, 10-13. [CrossRef]

21. Castellon, X.; Bogdanova, V. Chronic Inflammatory Diseases and Endothelial Dysfunction. Aging Dis. 2016, 7, 81-89. [CrossRef] [PubMed]

22. Hotamisligil, G.S. Inflammation and metabolic disorders. Nature 2006, 444, 860-867. [CrossRef] [PubMed]

23. Ross, R. Atherosclerosis-An Inflammatory Disease. N. Engl. J. Med. 1999, 340, 115-126. [CrossRef] [PubMed]

24. Steyers, C.M.; Miller, J.F.J. Endothelial Dysfunction in Chronic Inflammatory Diseases. Int. J. Mol. Sci. 2014, 15, 11324-11349. [CrossRef] [PubMed]

25. Ridker, P.M.; Everett, B.M.; Thuren, T.; MacFadyen, J.G.; Chang, W.H.; Ballantyne, C.; Fonseca, F.; Nicolau, J.; Koenig, W.; Anker, S.D.; et al. Antiinflammatory Therapy with Canakinumab for Atherosclerotic Disease. N. Engl. J. Med. 2017, 377, 1119-1131. [CrossRef] [PubMed]

26. GBD 2017 Diet Collaborators. Health effects of dietary risks in 195 countries, 1990-2017: A systematic analysis for the Global Burden of Disease Study 2017. Lancet 2019, 393, 1958-1972. [CrossRef]

27. Rahman, I.; Biswas, S.K.; Kirkham, P.A. Regulation of inflammation and redox signaling by dietary polyphenols. Biochem. Pharmacol. 2006, 72, 1439-1452. [CrossRef]

28. Yahfoufi, N.; Alsadi, N.; Jambi, M.; Matar, C. The Immunomodulatory and Anti-Inflammatory Role of Polyphenols. Nutrients 2018, 10, 1618. [CrossRef]

29. Fresco, P.; Borges, F.I.G.M.; Diniz, C.G.; Marques, M. New insights on the anticancer properties of dietary polyphenols. Med. Res. Rev. 2006, 26, 747-766. [CrossRef] [PubMed]

30. Weatherill, A.R.; Lee, J.Y.; Zhao, L.; Lemay, D.G.; Youn, H.S.; Hwang, D.H. Saturated and Polyunsaturated Fatty Acids Reciprocally Modulate Dendritic Cell Functions Mediated through TLR4. J. Immunol. 2005, 174, 5390-5397. [CrossRef] [PubMed]

31. Hotamisligil, G.S.; Erbay, E. Nutrient sensing and inflammation in metabolic diseases. Nat. Rev. Immunol. 2008, 8, 923-934. [CrossRef]

32. Rogero, M.M.; Calder, P.C. Obesity, Inflammation, Toll-Like Receptor 4 and Fatty Acids. Nutrients 2018, 10, 432. [CrossRef] [PubMed]

33. Childs, C.E.; Calder, P.C.; Miles, E.A. Diet and Immune Function. Nutrients 2019, 11, 1933. [CrossRef] [PubMed]

34. Pendyala, S.; Walker, J.M.; Holt, P.R. A High-Fat Diet Is Associated with Endotoxemia That Originates from the Gut. Gastroenterology 2012, 142, 1100-1101. [CrossRef] [PubMed]

35. Reedy, J.; Subar, A.F.; George, S.M.; Krebs-Smith, S.M. Extending Methods in Dietary Patterns Research. Nutrients 2018, 10 , 571. [CrossRef] [PubMed]

36. Dietary Guidelines Advisory Committee. Scientific Report of the 2015 Dietary Guidelines Advisory Committee. Available online: https:/ / health.gov/sites/default/ files/2019-09/Scientific-Report-of-the-2015-Dietary-Guidelines-Advisory-Committee. pdf (accessed on 4 May 2020).

37. Hlebowicz, J.; Persson, M.; Gullberg, B.; Sonestedt, E.; Wallström, P.; Drake, I.; Nilsson, J.; Hedblad, B.; Wirfält, E. Food patterns, inflammation markers and incidence of cardiovascular disease: The Malmö Diet and Cancer study. J. Intern. Med. 2011, 270, 365-376. [CrossRef] [PubMed]

38. De Mello, V.D.F.; Schwab, U.; Kolehmainen, M.; Koenig, W.; Siloaho, M.; Poutanen, K.; Mykkänen, H.; Uusitupa, M. A diet high in fatty fish, bilberries and wholegrain products improves markers of endothelial function and inflammation in individuals with impaired glucose metabolism in a randomised controlled trial: The Sysdimet study. Diabetology 2011, 54, 2755-2767. [CrossRef]

39. Berild, A.; Holven, K.B.; Ulven, S.M. Anbefalt nordisk kosthold og risikomarkører for hjerte- og karsykdom. Tidsskr. Nor. Leg. 2017, 137, 721-726. [CrossRef] [PubMed]

40. Bonaccio, M.; Di Castelnuovo, A.; De Curtis, A.; Costanzo, S.; Bracone, F.; Persichillo, M.; Donati, M.B.; De Gaetano, G.; Iacoviello, L. Nut consumption is inversely associated with both cancer and total mortality in a Mediterranean population: Prospective results from the Molisani study. Br. J. Nutr. 2015, 114, 804-811. [CrossRef]

41. Estruch, R.; Ros, E.; Salas-Salvadó, J.; Covas, M.-I.; Corella, D.; Arós, F.; Gómez-Gracia, E.; Ruiz-Gutiérrez, V.; Fiol, M.; Lapetra, J.; et al. Primary Prevention of Cardiovascular Disease with a Mediterranean Diet Supplemented with Extra-Virgin Olive Oil or Nuts. N. Engl. J. Med. 2018, 378, e34. [CrossRef] [PubMed]

42. Kanerva, N.; Loo, B.-M.; Eriksson, J.G.; Leiviskä, J.; Kaartinen, N.E.; Jula, A.; Männistö, S. Associations of the Baltic Sea diet with obesity-related markers of inflammation. Ann. Med. 2014, 46, 90-96. [CrossRef] [PubMed]

43. Esmaillzadeh, A.; Kimiagar, M.; Mehrabi, Y.; Azadbakht, L.; Hu, F.B.; Willett, W.C. Dietary Patterns and Markers of Systemic Inflammation among Iranian Women. J. Nutr. 2007, 137, 992-998. [CrossRef]

44. Vissers, L.; Waller, M.; Van Der Schouw, Y.; Hébert, J.; Shivappa, N.; Schoenaker, D.; Mishra, G. A pro-inflammatory diet is associated with increased risk of developing hypertension among middle-aged women. Nutr. Metab. Cardiovasc. Dis. 2017, 27, 564-570. [CrossRef] [PubMed] 
45. Ramallal, R.; Toledo, E.; Martinez-Gonzalez, M.; Hernandez-Hernandez, A.; Garcia-Arellano, A.; Shivappa, N.; Hebert, J.R.; Ruiz-Canela, M. Dietary Inflammatory Index and Incidence of Cardiovascular Disease in the SUN Cohort. PLoS ONE 2015, 10, e0135221. [CrossRef] [PubMed]

46. Spadafranca, A.; Piuri, G.; Bulfoni, C.; Liguori, I.; Battezzati, A.; Bertoli, S.; Speciani, A.F.; Ferrazzi, E. Adherence to the Mediterranean Diet and Serum Adiponectin Levels in Pregnancy: Results from a Cohort Study in Normal Weight Caucasian Women. Nutrients 2018, 10, 928. [CrossRef]

47. Renault, K.M.; Carlsen, E.M.; Hædersdal, S.; Nilas, L.; Secher, N.J.; Eugen-Olsen, J.; Cortes, D.; Olsen, S.F.; Halldorsson, T.I.; Norgaard, K. Impact of lifestyle intervention for obese women during pregnancy on maternal metabolic and inflammatory markers. Int. J. Obes. 2017, 41, 598-605. [CrossRef]

48. Effective Public Healthcare Panacea Project. Quality Assessment Tool for Quantitative Studies. Available online: https:/ www. ephpp.ca/quality-assessment-tool-for-quantitative-studies / (accessed on 15 June 2020).

49. McCullough, L.E.; Miller, E.E.; Calderwood, L.E.; Shivappa, N.; Steck, S.E.; Forman, M.R.; Mendez, M.A.; Maguire, R.; Fuemmeler, B.F.; Kollins, S.H.; et al. Maternal inflammatory diet and adverse pregnancy outcomes: Circulating cytokines and genomic imprinting as potential regulators? Epigenetics 2017, 12, 688-697. [CrossRef]

50. Lindsay, K.L.; Buss, C.; Wadhwa, P.D.; Entringer, S. Maternal Stress Potentiates the Effect of an Inflammatory Diet in Pregnancy on Maternal Concentrations of Tumor Necrosis Factor Alpha. Nutrients 2018, 10, 1252. [CrossRef] [PubMed]

51. Moore, B.F.; Sauder, K.A.; Starling, A.P.; Hébert, J.R.; Shivappa, N.; Ringham, B.M.; Glueck, D.H.; Dabelea, D. Proinflammatory Diets during Pregnancy and Neonatal Adiposity in the Healthy Start Study. J. Pediatr. 2018, 195, 121-127. [CrossRef]

52. Sen, S.; Rifas-Shiman, S.L.; Shivappa, N.; Wirth, M.D.; Hebert, J.R.; Gold, D.R.; Gillman, M.W.; Oken, E. Dietary Inflam-matory Potential during Pregnancy is Associated with Lower Fetal Growth and Breastfeeding Failure: Results from Project Viva. J. Nutr. 2016, 146, 728. [CrossRef]

53. Shin, D.; Hur, J.; Cho, E.H.; Chung, H.K.; Shivappa, N.; Wirth, M.D.; Hebert, J.R.; Lee, K.W. Pre-Pregnancy Body Mass In-dex Is Associated with Dietary Inflammatory Index and C-Reactive Protein Concentrations during Pregnancy. Nutrients 2017,9 , 351. [CrossRef] [PubMed]

54. Asemi, Z.; Samimi, M.; Tabassi, Z.; Sabihi, S.-S.; Esmaillzadeh, A. A randomized controlled clinical trial investigating the effect of DASH diet on insulin resistance, inflammation, and oxidative stress in gestational diabetes. Nutrients 2013, 29, 619-624. [CrossRef]

55. Vahid, F.; Shivappa, N.; Hekmatdoost, A.; Hebert, J.R.; Davoodi, S.H.; Sadeghi, M. Association between Maternal Dietary Inflammatory Index (DII) and abortion in Iranian women and validation of DII with serum concentration of inflammatory factors: Case-control study. Appl. Physiol. Nutr. Metab. 2017, 42, 511-516. [CrossRef]

56. Yang, Y.; Kan, H.; Yu, X.; Yang, Y.; Li, L.; Zhao, M. Relationship between dietary inflammatory index, hs-CRP level in the second trimester and neonatal birth weight: A cohort study. J. Clin. Biochem. Nutr. 2020, 66, 163-167. [CrossRef] [PubMed]

57. Papazian, T.; Serhal, A.; Hout, H.; Younes, H.; Tayeh, G.A.; Azouri, J.; Lteif, F.H.M.; Kesrouani, A.; Khabbaz, L.R. Discrepancies among different tools evaluating Mediterranean diet adherence during pregnancy, correlated to maternal anthropometric, dietary and biochemical characteristics. Clin. Nutr. 2019, 38, 1398-1405. [CrossRef]

58. García-Rodríguez, C.E.; Olza, J.; Aguilera, C.M.; Mesa, M.D.; Miles, E.A.; Noakes, P.S.; Vlachava, M.; Kremmyda, L.-S.; Diaper, N.D.; Godfrey, K.M.; et al. Plasma Inflammatory and Vascular Homeostasis Biomarkers Increase During Human Pregnancy but Are Not Affected by Oily Fish Intake. J. Nutr. 2012, 142, 1191-1196. [CrossRef] [PubMed]

59. Khoury, J.; Henriksen, T.; Seljeflot, I.; Morkrid, L.; Froslie, K.F.; Tonstad, S. Effects of an antiatherogenic diet during pregnan-cy on markers of maternal and fetal endothelial activation and inflammation: The CARRDIP study. BJOG 2007, 114, 279. [CrossRef]

60. Hrolfsdottir, L.; Schalkwijk, C.G.; Birgisdottir, B.E.; Gunnarsdottir, I.; Maslova, E.; Granström, C.; Strøm, M.; Olsen, S.F.; Halldorsson, T.I. Maternal diet, gestational weight gain, and inflammatory markers during pregnancy. Obesity 2016, 24, 2133-2139. [CrossRef] [PubMed]

61. Markovic, T.P.; Muirhead, R.; Overs, S.; Ross, G.P.; Louie, J.C.Y.; Kizirian, N.; Denyer, G.; Petocz, P.; Hyett, J.; Brand-Miller, J.C. Randomized Controlled Trial Investigating the Effects of a Low-Glycemic Index Diet on Pregnancy Outcomes in Women at High Risk of Gestational Diabetes Mellitus: The GI Baby 3 Study. Diabetes Care 2015, 39, 31-38. [CrossRef]

62. Röytiö, H.; Mokkala, K.; Vahlberg, T.; Laitinen, K. Dietary intake of fat and fibre according to reference values relates to higher gut microbiota richness in overweight pregnant women. Br. J. Nutr. 2017, 118, 343-352. [CrossRef]

63. Walsh, J.M.; Mahony, R.M.; Culliton, M.; Foley, M.E.; McAuliffe, F.M. Impact of a Low Glycemic Index Diet in Pregnancy on Markers of Maternal and Fetal Metabolism and Inflammation. Reprod. Sci. 2014, 21, 1378-1381. [CrossRef] [PubMed]

64. Locke, A.; Schneiderhan, J.; Zick, S.M. Diets for Health: Goals and Guidelines. American family physician 2018, 97, 721-728.

65. Cena, H.; Calder, P.C. Defining a Healthy Diet: Evidence for the Role of Contemporary Dietary Patterns in Health and Disease. Nutrients 2020, 12, 334. [CrossRef]

66. Miles, E.A.; Noakes, P.S.; Kremmyda, L.-S.; Vlachava, M.; Diaper, N.D.; Rosenlund, G.; Urwin, H.; Yaqoob, P.; Rossary, A.; Farges, M.-C.; et al. The Salmon in Pregnancy Study: Study design, subject characteristics, maternal fish and marine n-3 fatty acid intake, and marine n-3 fatty acid status in maternal and umbilical cord blood. Am. J. Clin. Nutr. 2011, 94, 1986S-1992S. [CrossRef] [PubMed]

67. Sichieri, R.; Everhart, J. Validity of a Brazilian food frequency questionnaire against dietary recalls and estimated energy intake. Nutr. Res. 1998, 18, 1649-1659. [CrossRef] 
68. Esfahani, F.H.; Asghari, G.; Mirmiran, P.; Azizi, F. Reproducibility and Relative Validity of Food Group Intake in a Food Frequency Questionnaire Developed for the Tehran Lipid and Glucose Study. J. Epidemiol. 2010, 20, 150-158. [CrossRef]

69. Papazian, T.; Hout, H.; Sibai, D.; Helou, N.; Younes, H.; El Osta, N.; Khabbaz, L.R. Development, reproducibility and validity of a food frequency questionnaire among pregnant women adherent to the Mediterranean dietary pattern. Clin. Nutr. 2016, 35, 1550-1556. [CrossRef]

70. Scholl, T.O.; Chen, X.; Khoo, C.S.; Lenders, C. The Dietary Glycemic Index during Pregnancy: Influence on Infant Birth Weight, Fetal Growth, and Biomarkers of Carbohydrate Metabolism. Am. J. Epidemiol. 2004, 159, 467-474. [CrossRef] [PubMed]

71. Fawzi, W. Calibration of a semi-quantitative food frequency questionnaire in early pregnancy. Ann. Epidemiol. 2004, 14, 754-762. [CrossRef] [PubMed]

72. Olsen, S.F.; Hansen, H.S.; Sandström, B.; Jensen, B. Erythrocyte levels compared with reported dietary intake of marine n-3 fatty acids in pregnant women. Br. J. Nutr. 1995, 73, 387-395. [CrossRef] [PubMed]

73. Johnson, C.L.; Paulose-Ram, R.; Ogden, C.L.; Carroll, M.D.; Kruszon-Moran, D.; Dohrmann, S.M.; Curtin, L.R. National health and nutrition examination survey: Analytic guidelines, 1999-2010. Vital Health Stat. Ser. 2 2013, 2, 1-24.

74. Walsh, J.; Mahony, R.; Foley, M.; Mc Auliffe, F. A randomised control trial of low glycaemic index carbohydrate diet versus no dietary intervention in the prevention of recurrence of macrosomia. BMC Pregnancy Childbirth 2010, 10, 16. [CrossRef] [PubMed]

75. Effective Public Health Practice Project. Quality Assessment Tool for Quantitative Studies Dictionary. Available online: https: / / www.ephpp.ca/PDF/QADictionary_dec2009.pdf (accessed on 15 June 2020).

76. Venn, B.J.; Green, T.J. Glycemic index and glycemic load: Measurement issues and their effect on diet-disease relationships. Eur. J. Clin. Nutr. 2007, 61, S122-S131. [CrossRef] [PubMed]

77. Willett, W. Nutritional Epidemiology; Oxford University Press: Oxford, UK, 2013.

78. Shivappa, N.; Steck, S.E.; Hurley, T.G.; Hussey, J.R.; Hébert, J.R. Designing and developing a literature-derived, population-based dietary inflammatory index. Public Health Nutr. 2014, 17, 1689-1696. [CrossRef] [PubMed]

79. Hernandez, T.L.; Van Pelt, R.E.; Anderson, M.A.; Reece, M.S.; Reynolds, R.M.; De La Houssaye, B.A.; Heerwagen, M.; Donahoo, W.T.; Daniels, L.J.; Chartier-Logan, C.; et al. Women with Gestational Diabetes Mellitus Randomized to a Higher-Complex Carbohydrate/Low-Fat Diet Manifest Lower Adipose Tissue Insulin Resistance, Inflammation, Glucose, and Free Fatty Acids: A Pilot Study. Diabetes Care 2015, 39, 39-42. [CrossRef] [PubMed]

80. Castell, J.V.; Gómez-Lechón, M.J.; David, M.; Andus, T.; Geiger, T.; Trullenque, R.; Fabra, R.; Heinrich, P.C. Interleukin-6 is the major regulator of acute phase protein synthesis in adult human hepatocytes. FEBS Lett. 1989, 242, 237-239. [CrossRef]

81. Calder, P.C.; Ahluwalia, N.; Albers, R.; Bosco, N.; Bourdet-Sicard, R.; Haller, D.; Holgate, S.; Jönsson, L.; Latulippe, M.; Marcos, A.; et al. A Consideration of Biomarkers to be Used for Evaluation of Inflammation in Human Nutritional Studies. Br. J. Nutr. 2013, 109, S1-S34. [CrossRef] [PubMed]

82. Shim, J.-S.; Oh, K.; Kim, H.C. Dietary assessment methods in epidemiologic studies. Epidemiol. Health 2014, 36 , e2014009. [CrossRef] [PubMed]

83. Clendenen, T.V.; Koenig, K.L.; Arslan, A.A.; Lukanova, A.; Berrino, F.; Gu, Y.; Hallmans, G.; Idahl, A.; Krogh, V.; Lokshin, A.E.; et al. Factors associated with inflammation markers, a cross-sectional analysis. Cytokine 2011, 56, 769-778. [CrossRef] [PubMed]

84. Shiels, M.S.; Katki, H.A.; Freedman, N.D.; Purdue, M.P.; Wentzensen, N.; Trabert, B.; Kitahara, C.M.; Furr, M.; Li, Y.; Kemp, T.J.; et al. Cigarette Smoking and Variations in Systemic Immune and Inflammation Markers. J. Natl. Cancer Inst. 2014, 106. [CrossRef]

85. Calle, M.C.; Andersen, C.J. Assessment of Dietary Patterns Represents a Potential, Yet Variable, Measure of Inflammatory Status: A Review and Update. Dis. Markers 2019, 2019, 3102870. [CrossRef] [PubMed]

86. Calder, P.C.; Ahluwalia, N.; Brouns, F.; Buetler, T.; Clement, K.; Cunningham, K.; Esposito, K.; Jö Nsson, L.S.; Kolb, H.; Lansink, M.; et al. Dietary factors and low-grade inflammation in relation to overweight and obesity commissioned by the ILSI Europe Metabolic Syndrome and Diabetes Task Force. Br. J. Nutr. 2011, 106, S1-S78. [CrossRef]

87. Schulze, M.B.; A Martínez-González, M.; Fung, T.T.; Lichtenstein, A.H.; Forouhi, N.G. Food based dietary patterns and chronic disease prevention. BMJ 2018, 361, k2396. [CrossRef]

88. Staudacher, H.M.; Irving, P.M.; Lomer, M.C.E.; Whelan, K. The challenges of control groups, placebos and blinding in clinical trials of dietary interventions. Proc. Nutr. Soc. 2017, 76, 203-212. [CrossRef]

89. Tarasuk, V.S.; Brooker, A.-S. Interpreting epidemiologic studies of diet-disease relationships. J. Nutr. 1997, 127, 1847-1852. [CrossRef] [PubMed]

90. Minihane, A.M.; Vinoy, S.; Russell, W.R.; Baka, A.; Roche, H.M.; Tuohy, K.M.; Teeling, J.L.; Blaak, E.E.; Fenech, M.; Vauzour, D.; et al. Low-grade inflammation, diet composition and health: Current research evidence and its translation. Br. J. Nutr. 2015, 114, 999-1012. [CrossRef]

91. Ferretti, F.; Mariani, M. Simple vs. Complex Carbohydrate Dietary Patterns and the Global Overweight and Obesity Pandemic. Int. J. Environ. Res. Public Health 2017, 14, 1174. [CrossRef] [PubMed]

92. Sears, B.; Ricordi, C. Anti-Inflammatory Nutrition as a Pharmacological Approach to Treat Obesity. J. Obes. 2010, $2011,431985$. [CrossRef]

93. Serhan, C.N.; Savill, J. Resolution of inflammation: The beginning programs the end. Nat. Immunol. 2005, 6, 1191-1197. [CrossRef] [PubMed] 
94. Serhan, C.N.; Brain, S.D.; Buckley, C.D.; Gilroy, D.W.; Haslett, C.; O’Neill, L.A.J.; Perretti, M.; Rossi, A.G.; Wallace, J.L. Resolution of in flammation: State of the art, definitions and terms. FASEB J. 2007, 21, 325-332. [CrossRef] [PubMed]

95. Serhan, C.N. Pro-resolving lipid mediators are leads for resolution physiology. Nature 2014, 510, 92-101. [CrossRef] [PubMed]

96. Keelan, J.A.; Mas, E.; D’Vaz, N.; Dunstan, J.A.; Li, S.; Barden, A.E.; Mark, P.J.; Waddell, B.J.; Prescott, S.L.; Mori, T.A. Effects of maternal n-3 fatty acid supplementation on placental cytokines, pro-resolving lipid mediators and their precursors. Reproduction 2015, 149, 171-178. [CrossRef] [PubMed]

97. Mozurkewich, E.L.; Greenwood, M.; Clinton, C.; Berman, D.; Romero, V.; Djuric, Z.; Qualls, C.; Gronert, K. Pathway Markers for Pro-resolving Lipid Mediators in Maternal and Umbilical Cord Blood: A Secondary Analysis of the Mothers, Omega-3, and Mental Health Study. Front. Pharmacol. 2016, 7, 274. [CrossRef] [PubMed] 